\title{
Pandemia COVID-19: efeitos do distanciamento social no comportamento alimentar de estudantes do estado do Ceará
}

COVID-19 Pandemic: effects of social distancing on the eating behavior of students in the state of Ceará

Pandemia COVID-19: efectos del distanciamiento social en la conducta alimentaria de los estudiantes del estado de Ceará

Recebido: 07/08/2021 | Revisado: 14/08/2021 | Aceito: 19/08/2021 | Publicado: 22/08/2021

Leonel Francisco de Oliveira Freire

ORCID: https://orcid.org/0000-0002-8734-5292 Universidade do Estado do Rio Grande do Norte, Brasil E-mail: leonelloliveira93@gmail.com

Maria Aparecida Cursino

ORCID: https://orcid.org/0000-0001-9312-6056 Universidade Estadual de Campinas, Brasil E-mail: ma.cursino@gmail.com

Danielle Christina Lino Leal

ORCID: https://orcid.org/0000-0002-1048-3435 Universidade do Estado do Rio Grande do Norte, Brasil E-mail: danielleleal_@hotmail.com

Adriana de Freitas Morais

ORCID: https://orcid.org/0000-0003-3556-7499 Instituto Federal de Educação, Ciência e Tecnologia do Ceará, Brasil

E-mail: adriana.morais.nutricionista@gmail.com

Matheus da Silva Regis

ORCID: https://orcid.org/0000-0001-9402-6030 Universidade do Estado do Rio Grande do Norte, Brasil E-mail: matheussregis@gmail.com

Isabela Pinheiro Cavalcante Lima

ORCID: https://orcid.org/0000-0002-7681-9675 Universidade do Estado do Rio Grande do Norte, Brasil E-mail: belapcl@yahoo.com.br

Rayza Maria Silva Leal

ORCID: https://orcid.org/0000-0003-4305-8598 Faculdade de Ciências Humanas e da Saúde de Minas Gerais, Brasil E-mail: rayza.leal@hotmail.com

Marielen Ghedin da Silva

ORCID: https://orcid.org/0000-0001-8970-8563

Universidade do Extremo Sul Catarinense, Brasil

E-mail: ghedin.nutri@gmail.com

\begin{abstract}
Resumo
A pandemia causada pela COVID-19 afetou, em escala mundial, a rotina de diversos estudantes. Para este grupo, frequentemente acometido por transtornos mentais, o distanciamento social teve maior impacto na saúde mental. A necessidade de isolamento social impossibilitou a permanência das aulas presenciais, modificando hábitos sociais e alimentares. Nesse cenário, o estresse, o sofrimento psicológico e o sono irregular, atuam como agentes modificadores do comportamento alimentar, levando à adoção de condutas lesivas à saúde, como mecanismo compensatório. No entanto, as pesquisas voltadas a esse público ainda são escassas, diante disso, este estudo tem como objetivo avaliar o comportamento alimentar de estudantes de instituições de ensino público federal, situadas no interior do estado do Ceará. Metodologia: Os participantes da pesquisa responderam um questionário online autoaplicável contendo perguntas referentes ao consumo de vegetais, frutas, leite e iogurtes no período anterior e durante a pandemia. Resultados: Os achados revelaram uma elevação da frequência e consumo de vegetais, frutas, leites e iogurtes durante o período de distanciamento social. Conclusão: Neste estudo podemos identificar uma melhoria nos hábitos alimentares dos estudantes, sendo caracterizado pela elevação da frequência e consumo de vegetais e frutas, sob a forma de saladas cruas ou cozidas, bem como o consumo de leite e iogurtes. Essa modificação do padrão alimentar pode ser resultante da maior disponibilidade de tempo para o preparo de refeições, retorno ao ambiente familiar e a utilização da alimentação como ferramenta essencial para o fortalecimento da saúde e do sistema imunológico.
\end{abstract}

Palavras-chave: Comportamento alimentar; Hábitos alimentares; SARS-CoV-2; Estudantes. 


\begin{abstract}
The pandemic caused by COVID-19 affected, on a global scale, the routine of many students. For this group, often affected by mental disorders, social distancing had a greater impact on mental health. The need for social isolation made it impossible to maintain classroom classes, modifying social and eating habits. In this scenario, stress, psychological suffering and irregular sleep act as modifying agents of eating behavior, leading to the adoption of behaviors that are harmful to health, as a compensatory mechanism. However, research aimed at this audience is still scarce, therefore, this study aims to assess the eating behavior of students from federal public education institutions located in the interior of the state of Ceará. Methodology: Research participants answered a self-administered online questionnaire containing questions regarding the consumption of vegetables, fruits, milk and yogurts in the period before and during the pandemic. Results: The findings revealed an increase in the frequency and consumption of vegetables, fruits, milk and yogurts during the period of social distancing. Conclusion: In this study, we can identify an improvement in the students' eating habits, characterized by an increase in the frequency and consumption of vegetables and fruits, in the form of raw or cooked salads, as well as the consumption of milk and yogurt. This change in dietary patterns may result from the greater availability of time for preparing meals, returning to the family environment and the use of food as an essential tool for strengthening health and the immune system.
\end{abstract}

Keywords: Feeding behavior; Eating habits; SARS-CoV-2; Students.

\title{
Resumen
}

La pandemia provocada por COVID-19 afectó, a escala global, la rutina de muchos estudiantes. Para este grupo, a menudo afectado por trastornos mentales, el distanciamiento social tuvo un mayor impacto en la salud mental. La necesidad de aislamiento social imposibilitó el mantenimiento de las clases en el aula, modificando los hábitos sociales y alimentarios. En este escenario, el estrés, el sufrimiento psicológico y el sueño irregular actúan como agentes modificadores de la conducta alimentaria, conduciendo a la adopción de conductas nocivas para la salud, como mecanismo compensatorio. Sin embargo, la investigación dirigida a este público aún es escasa, por lo que este estudio tiene como objetivo evaluar la conducta alimentaria de estudiantes de instituciones de educación pública federal ubicadas en el interior del estado de Ceará. Metodología: Los participantes de la investigación respondieron un cuestionario en línea autoadministrado que contenía preguntas sobre el consumo de verduras, frutas, leche y yogures en el período anterior y durante la pandemia. Resultados: Los hallazgos revelaron un aumento en la frecuencia y el consumo de verduras, frutas, leche y yogures durante el período de distanciamiento social. Conclusión: En este estudio, podemos identificar una mejora en los hábitos alimentarios de los estudiantes, caracterizada por un aumento en la frecuencia y consumo de verduras y frutas, en forma de ensaladas crudas o cocidas, así como el consumo de leche y yogur. Este cambio en los patrones dietéticos puede resultar de la mayor disponibilidad de tiempo para la preparación de las comidas, el regreso al entorno familiar y el uso de la comida como herramienta fundamental para fortalecer la salud y el sistema inmunológico.

Palabras clave: Comportamiento alimentario; Hábitos alimenticios; SARS-CoV-2; Estudiantes.

\section{Introdução}

Os estudantes universitários e pré-universitários constituem um grupo com elevado acometimento por transtornos mentais, caracterizados, principalmente, por mudanças no humor e ansiedade. Essa fase é marcada por uma transição entre a adolescência e a fase adulta e, associada a esse evento, a mudança do nível acadêmico pode atuar como um catalizador para o desenvolvimento de transtorno mentais, tornando urgente a atenção especial a saúde mental desse grupo (Auerbach et al., 2018; Boukrim, Obtel, Kasouati, Achbani, \& Razine, 2021).

A pandemia causada pela COVID-19 afetou em escala mundial a rotina de diversos estudantes. A necessidade do distanciamento social impossibilitou a permanência das aulas presenciais impondo o ensino à distância como nova modalidade. Essa alteração gerou a modificação de hábitos sociais, devido ao fechamento de estabelecimentos como restaurantes, bares, shoppings e academias, locais frequentados amplamente pelo público jovem, impactando a saúde mental (Keel et al., 2020; Martínez-de-Quel, Suárez-Iglesias, López-Flores, \& Pérez, 2021).

O distanciamento social gera uma série de efeitos psicológicos negativos em pacientes com transtornos mentais préexistentes ou não. Dentre as alterações psicológicas e comportamentais, os transtornos alimentares ganharam ênfase nessa fase. O estresse, o sofrimento psicológico e o sono irregular, presentes em uma ampla parcela de pessoas, atuam como agentes modificadores do comportamento alimentar, levando ao consumo excessivo de bebidas açucaradas, alimentos ricos em calorias e ao aumento no número de lanches entre as refeições (Brooks et al., 2020; Coakley, Le, Silva, \& Wilks, 2021; De Pasquale et 
al., 2021; Poelman et al., 2021).

No Brasil poucas pesquisas foram realizadas investigando o comportamento alimentar de estudantes durante o período pandêmico. Quando tratamos do perfil de comportamentos de estudantes originários de cidades interioranas, a literatura científica é ainda mais escassa. Diante dessa lacuna científica, este estudo tem como objetivo avaliar o comportamento alimentar de estudantes universitários e do ensino técnico de instituições de ensino público federal, situadas no interior do estado do Ceará.

\section{Metodologia}

Os efeitos da quarentena no comportamento alimentar foram investigados através de uma pesquisa on-line, anônima, utilizando como ferramenta de investigação um questionário semiestruturado e autoaplicável, adaptado do VIGITEL (Ministério da Saúde, 2020). Essa abordagem de pesquisa é recomendada para estudos que visam alcançar um público específico em um pequeno período de tempo e em situações cujo distanciamento social impossibilita o contato com o público alvo (Sidor \& Rzymski, 2020).

Foi criado um questionário na plataforma online Google Forms ${ }^{\circledR}$ e disponibilizado o link de acesso em grupos de estudantes nas redes sociais durante os meses de maio e junho de 2020. Os critérios de inclusão na pesquisa foram: concordar com os termos da pesquisa, ser estudante do ensino técnico ou superior, estar regularmente matriculado durante o isolamento social e responder todas as perguntas do questionário. Objetivando reduzir os riscos de ordem moral e psicológica, as perguntas do questionário foram elaboradas garantindo o anonimato dos participantes, impossibilitando a identificação das respostas individuais. O questionário teve como objetivo avaliar o consumo e a frequência alimentar entre estudantes do ensino técnico e superior de duas instituições federais de ensino localizadas no interior do estado do Ceará.

A população do estudo foi composta por estudantes universitários e do ensino técnico de duas instituições federais de ensino localizadas no estado do Ceará, Brasil. A amostra foi do tipo probabilística aleatória. O cálculo amostral foi realizado utilizando-se o software Open Epi®, versão 3.01, e empregou-se a seguinte fórmula: n= N x z2 x P(1-P)/ d2 (N-1) + z2 x P(1P). Onde $\mathrm{n}=$ tamanho da amostra; $\mathrm{N}=$ população de referência (176); $\mathrm{z}=$ nível de confiança escolhido (95\%), desvio padrão $(1,8) ; \mathrm{P}=$ prevalência esperada do fenômeno a ser investigado, sendo considerado o valor de 4,2\% (Amatori et al., 2020); $\mathrm{d}=$ erro amostral previsto (5\%). Assim, o n calculado resultou em 45 indivíduos e, considerando um valor de perda amostral de $10 \%$, o n passou a ser representado por 55 indivíduos.

As respostas do formulário online foram exportadas para uma planilha do software Microsoft Excel®, versão 2013. Posteriormente, os dados foram transferidos para o software "IBM SPSS Statistics for Windows", versão 25.0, e executada a análise estatística.

A análise estatística baseou-se na frequência semanal do consumo de alimentos. As perguntas do questionário avaliavam a frequência alimentar semanal tendo como base as seguintes categorias: 1 a 2 vezes, 3 a 4 vezes, 5 a 6 vezes, Todos os dias e Não consome. As frequências encontradas foram comparadas em dois momentos, o anterior a pandemia e durante a pandemia. A análise foi realizada através da aplicação do teste qui-quadrado, que se baseia na avaliação dos resultados de um estudo e os valores de distribuição esperadas. Foi considerado um grau de confiança de $95 \%$ e a diferença estatisticamente significativa quando $\mathrm{p}<0,05$. Os valores de qui-quadrado foram interpretados de acordo com Donald, 2015.

\section{Resultados}

O formulário online foi divulgado em grupos de redes sociais de duas instituições de ensino superior e técnico localizadas no interior do estado do Ceará nos meses de julho e agosto de 2020. Conforme a Tabela 1, a amostra do estudo foi composta por 106 estudantes que responderam ao questionário online autoaplicável. Dos participantes, 59,4\% eram do sexo 
feminino, na faixa etária de 18 a 24 anos (50,9\%), solteiros (78,3\%), declarando renda de até um salário mínimo (46,2\%) e ensino superior incompleto $(41,5 \%)$.

Tabela 1: Caracterização da amostra quanto ao sexo, idade, estado civil, renda e escolaridade.

\begin{tabular}{llll}
\hline Sexo & & $\mathbf{n}$ & $\mathbf{\%}$ \\
\hline \multirow{3}{*}{ Idade } & Masculino & 43 & 40,6 \\
& Feminino & 63 & 59,4 \\
& Até 17 anos & 3 & 2,8 \\
& 18 a 24 anos & 54 & 50,9 \\
Estado Civil & 25 a 35 anos & 36 & 34,0 \\
& 36 a 50 anos & 9 & 8,5 \\
\multirow{3}{*}{ Renda } & Acima de 50 anos & 4 & 3,8 \\
& Solteiro & 83 & 78,3 \\
& Casado & 20 & 18,3 \\
Escolaridade & Outros & 3 & 2,8 \\
& Até 1 salário mínimo & 49 & 46,2 \\
& 2 a 3 salários mínimos & 34 & 32,1 \\
& Acima de 3 salários mínimos & 23 & 21,7 \\
& Ensino Médio Incompleto & 2 & 1,9 \\
& Ensino Médio Completo & 12 & 11,3 \\
& Ensino Superior Incompleto & 44 & 41,5 \\
& Ensino Superior Completo & 12 & 11,3 \\
& Pós-Graduação & 36 & 34,0 \\
\hline
\end{tabular}

Fonte: Autores (2021).

Quando comparadas as frequências de consumo de salada crua antes e durante a pandemia, pode-se inferir que, com o distanciamento social, houve um aumento de $2,9 \%$ no consumo de saladas cruas entre as pessoas que relataram consumir 1 ou 2 vezes por semana ou todos os dias. Já quando a frequência era de 3 a 4 vezes por semana e de 5 a 6 vezes por semana, houve uma redução de 3,8\% e 1,9\% no consumo, respectivamente. O percentual de frequência entre os que não consumiam salada crua manteve-se igual nos dois momentos (Figura 1).

A frequência do consumo de salada cozida aumentou discretamente durante o período pandêmico. O aumento foi de $1,8 \%$, entre os indivíduos que consumiam 1 a 2 vezes por semana e de 1,9\% entre os que consumiam 5 a 6 vezes por semana. Os que declararam consumir a salada cozida de 3 a 4 vezes por semana, diminuíram a frequência da ingestão em 3,8\%. Da mesma forma que ao encontrado no consumo de salada crua, a frequência do consumo diário para os que não consumiam salada cozida permaneceu sem alterações (Figura 2). 
Figura 1: Frequência do consumo de salada crua antes e durante a pandemia.

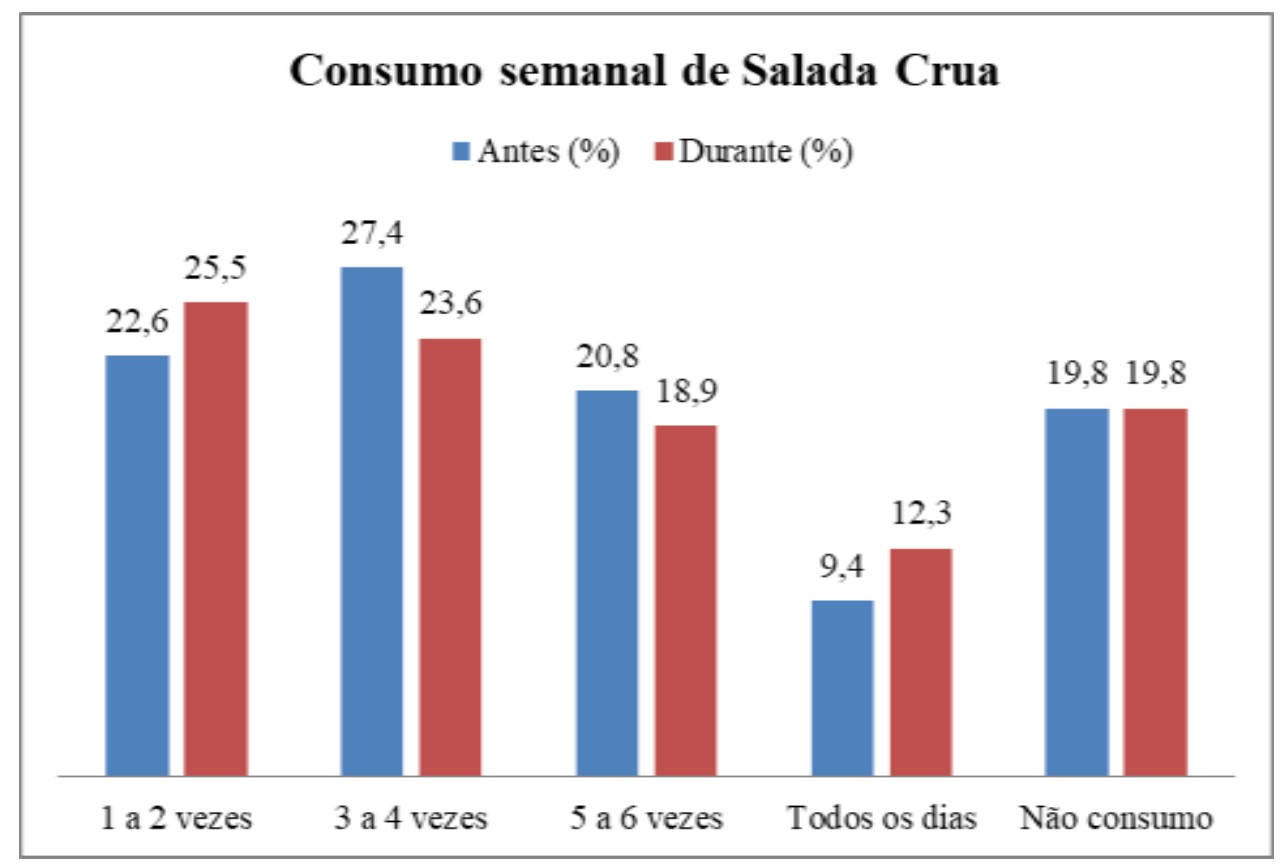

Fonte: Autores (2021).

Figura 2: Frequência do consumo de legumes e verduras cozidas antes e durante a pandemia.

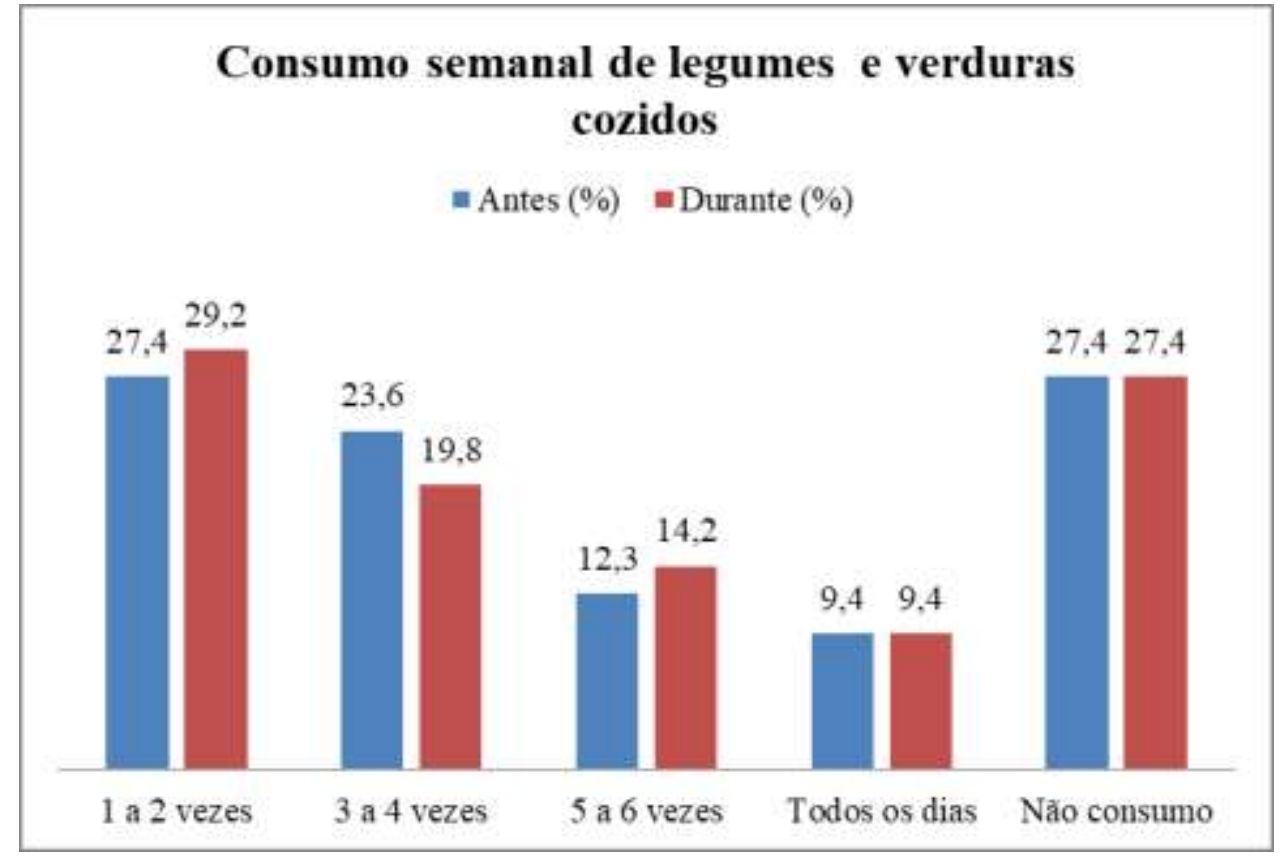

Fonte: Autores (2021).

Ao analisar a frequência do consumo de frutas frescas ou saladas de frutas antes e durante a pandemia, percebeu-se que $3,8 \%$ da amostra passou a consumi-las durante a pandemia, passando de 10,4\% que não consumiam, para 6,6\%. Houve, ainda, um aumento de 5,7\% de pessoas que passaram a consumi-las de 3 a 4 vezes por semana. 
Figura 3: Frequência do consumo de frutas frescas ou salada de frutas antes e durante a pandemia.

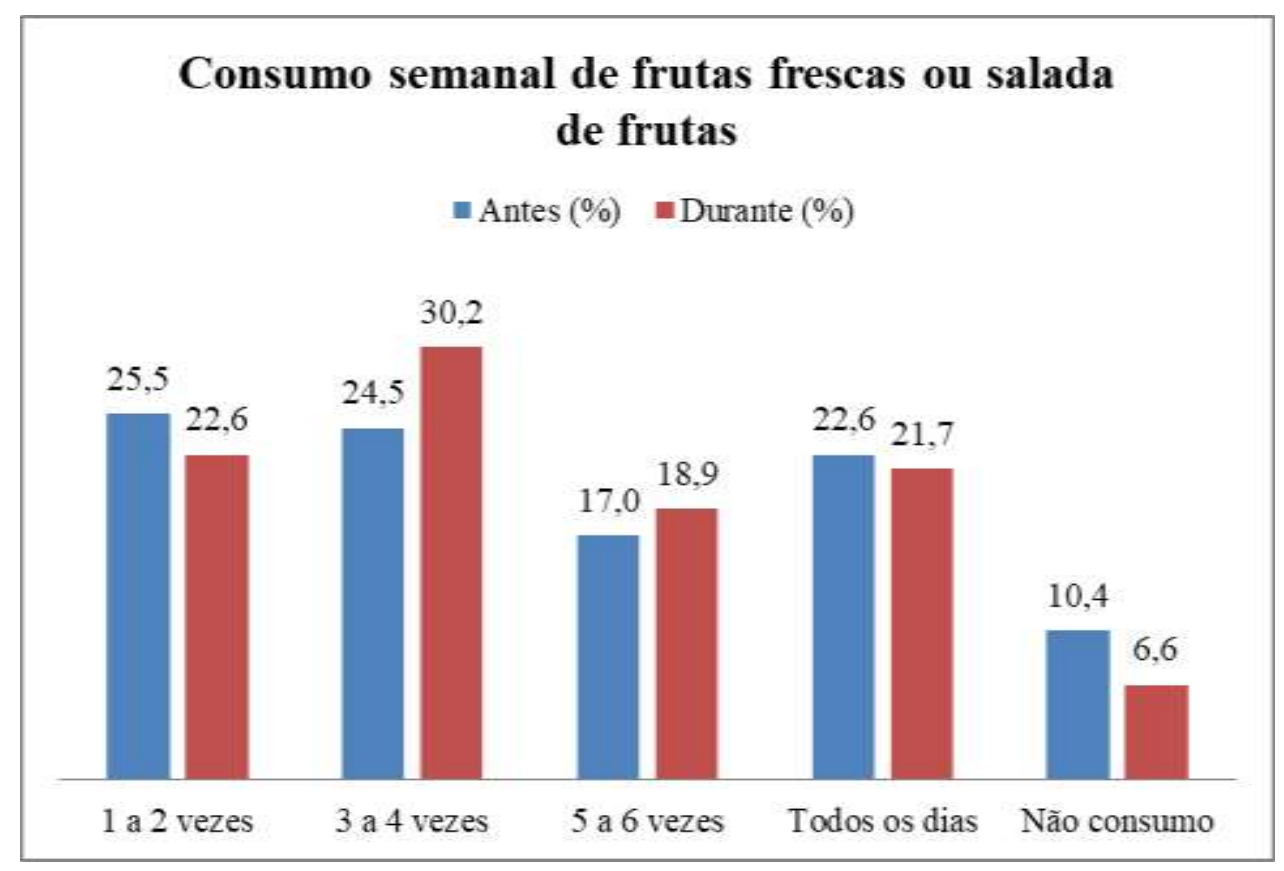

Fonte: Autores (2021).

O consumo de leite ou iogurtes durante o distanciamento também aumentou, o aumento foi de 4,7\%. Dentre os participantes 4,7\% passaram a consumir leite ou iogurtes, totalizando 26,4\% da amostra. Além disso, o consumo diário também teve uma elevação de $1,9 \%$. Os achados revelam também uma redução da frequência na faixa de 1 a 2 dias por semana, reforçando o aumento do consumo no período. Ver Figura 4.

Figura 4: Frequência do consumo de leite ou iogurte antes e durante a pandemia.

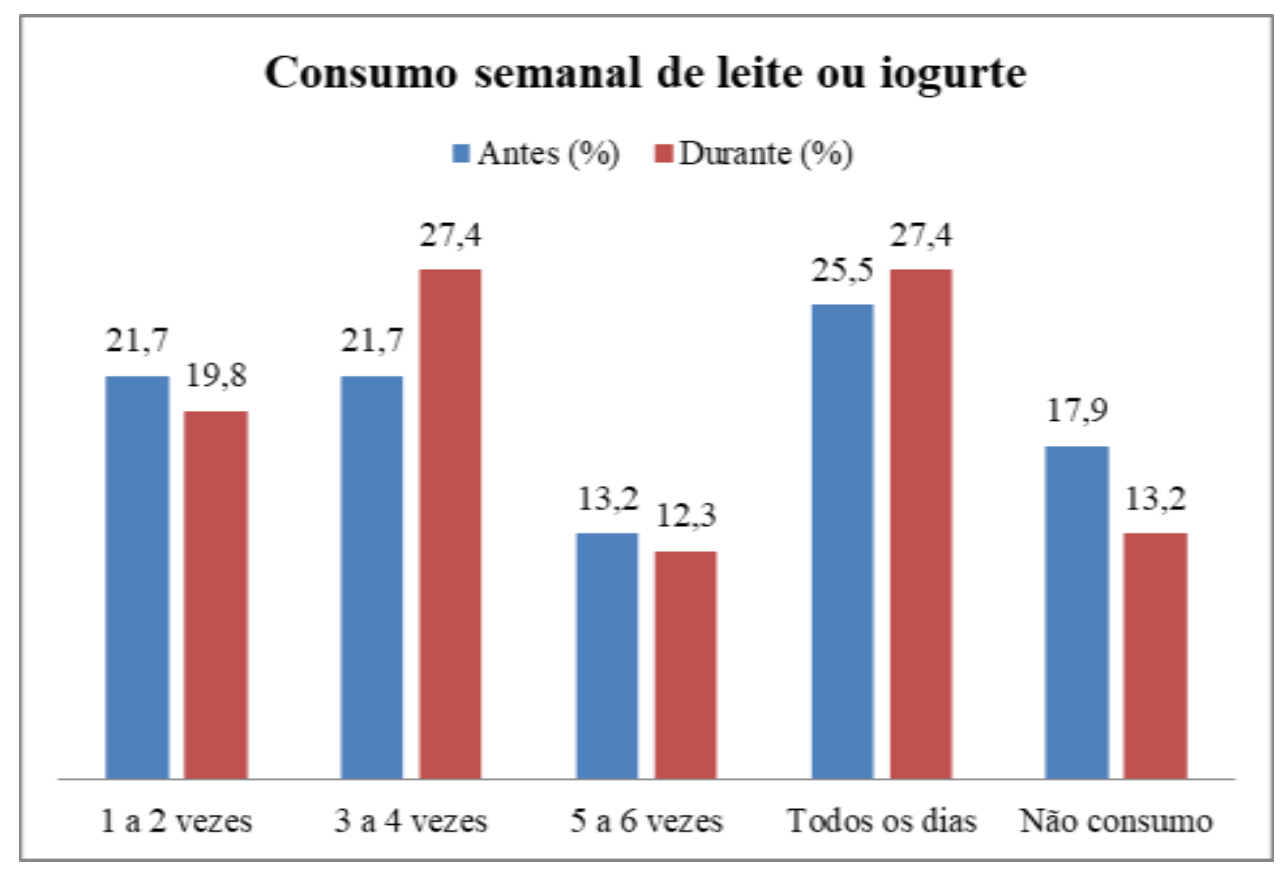

Fonte: Autores (2021).

A análise inferencial realizada através do teste do Qui-quadrado mostrou uma diferença estatística significativa 
$(\mathrm{p}<0,005)$ entre os dois momentos, anterior a pandemia e durante a pandemia, para o consumo de salada crua, legumes e verduras cozidos, frutas frescas ou saladas de frutas e leite ou iogurte. Conforme apresentado na Tabela 2.

Tabela 2: Teste Qui-quadrado para a frequência do consumo alimentar nos períodos antes e durante a pandemia da Covid 19.

\begin{tabular}{|c|c|c|c|c|c|c|c|c|c|c|c|c|c|}
\hline \multirow{3}{*}{\multicolumn{3}{|c|}{$\begin{array}{l}\text { Distribuição de } \\
\text { frequências } \\
\text { Antes da pandemia }\end{array}$}} & \multicolumn{11}{|c|}{ Durante a pandemia } \\
\hline & & & \multirow{2}{*}{\multicolumn{2}{|c|}{1 a 2 vezes }} & \multicolumn{6}{|c|}{ Consumo semanal de Salada crua } & \multirow{2}{*}{\multicolumn{2}{|c|}{ NC }} & \multirow{3}{*}{$\begin{array}{c}\mathbf{p} \\
0,001\end{array}$} \\
\hline & & & & & $3 \mathbf{a}$ & vezes & $5 \mathbf{a}$ & vezes & & TD & & & \\
\hline & $\mathbf{n}$ & $\%$ & $\mathbf{n}$ & $\%$ & $\mathbf{n}$ & $\%$ & $\mathbf{n}$ & $\%$ & n & $\%$ & $\mathbf{n}$ & $\%$ & \\
\hline $\begin{array}{l}1 \text { a } 2 \text { vezes } \\
\text { por semana }\end{array}$ & 24 & 22,6 & 18 & 75,0 & 3 & 12,5 & 0,0 & 0,0 & 1 & 0,0 & 2 & 8,3 & \\
\hline $\begin{array}{l}3 \text { a } 4 \text { vezes } \\
\text { por semana }\end{array}$ & 29 & 27,4 & 3 & 10,3 & 19 & 65,5 & 4 & 13,8 & 0 & 0,0 & 3 & 10,3 & \\
\hline $\begin{array}{l}5 \text { a } 6 \text { vezes } \\
\text { por semana }\end{array}$ & 22 & 20,8 & 2 & 9,1 & 3 & 13,6 & 13 & 59,1 & 2 & 9,1 & 2 & 9,1 & \\
\hline Todo dia & 10 & 9,4 & 0 & 0,0 & 0 & 0,0 & 0 & 0,0 & 10 & 100,0 & 0 & 0,0 & \\
\hline \multirow{2}{*}{$\begin{array}{l}\text { Não } \\
\text { Consome }\end{array}$} & 21 & 19,8 & 4 & 19,0 & 0 & 0,0 & 3 & 14,3 & 0 & 0,0 & 14 & 66,7 & \\
\hline & & & \multicolumn{11}{|c|}{ Consumo semanal de legumes e verduras cozidos } \\
\hline $\begin{array}{l}1 \text { a } 2 \text { vezes } \\
\text { por semana }\end{array}$ & 29 & 27,4 & 20 & 69,0 & 3 & 10,3 & 2 & 6,9 & 0 & 0,0 & 4 & 13,8 & 0,001 \\
\hline $\begin{array}{l}3 \text { a } 4 \text { vezes } \\
\text { por semana }\end{array}$ & 25 & 23,6 & 5 & 20,0 & 16 & 64,0 & 3 & 12,0 & 0 & 0,0 & 1 & 4,0 & \\
\hline $\begin{array}{l}5 \text { a } 6 \text { vezes } \\
\text { por semana }\end{array}$ & 13 & 12,3 & 1 & 7,7 & 2 & 15,4 & 9 & 69,2 & 1 & 7,7 & 0 & 0,0 & \\
\hline Todo dia & 10 & 9,4 & 1 & 10,0 & 0 & 0,0 & 0 & 0,0 & 9 & 90,0 & 0 & 0,0 & \\
\hline \multirow{2}{*}{$\begin{array}{l}\text { Não } \\
\text { Consome }\end{array}$} & 29 & 27,4 & 4 & 13,8 & 0 & 0,0 & 1 & 3,4 & 0 & 0,0 & 24 & 82,8 & \\
\hline & & & \multicolumn{10}{|c|}{ Consumo semanal de frutas frescas ou salada de frutas } & \\
\hline $\begin{array}{l}1 \text { a } 2 \text { vezes } \\
\text { por semana }\end{array}$ & 27 & 25,5 & 14 & 51,9 & 7 & 25,9 & 4 & 14,8 & 1 & 3,7 & 1 & 3,7 & 0,001 \\
\hline $\begin{array}{l}3 \text { a } 4 \text { vezes } \\
\text { por semana }\end{array}$ & 26 & 24,5 & 4 & 15,4 & 18 & 69,2 & 4 & 15,4 & 0 & 0,0 & 0 & 0,0 & \\
\hline $\begin{array}{l}5 \text { a } 6 \text { vezes } \\
\text { por semana }\end{array}$ & 18 & 17,0 & 2 & 11,1 & 5 & 27,8 & 10 & 55,6 & 1 & 5,6 & 0 & 0,0 & \\
\hline Todo dia & 24 & 22,6 & 0 & 0,0 & 1 & 4,2 & 2 & 8,3 & 21 & 87,5 & 0 & 0,0 & \\
\hline \multirow{2}{*}{$\begin{array}{l}\text { Não } \\
\text { Consome }\end{array}$} & 11 & 10,4 & 4 & 36,4 & 1 & 9,1 & 0 & 0,0 & 0 & 0,0 & 6 & 54,5 & \\
\hline & & \multicolumn{12}{|c|}{ Consumo semanal de leite ou iogurte } \\
\hline $\begin{array}{l}1 \text { a } 2 \text { vezes } \\
\text { por semana }\end{array}$ & 23 & 21,7 & 14 & 60,9 & 8 & 34,8 & 0 & 0,0 & 1 & 4,3 & 0 & 0,0 & 0,001 \\
\hline $\begin{array}{l}3 \text { a } 4 \text { vezes } \\
\text { por semana }\end{array}$ & 23 & 21,7 & 4 & 17,4 & 14 & 60,9 & 4 & 17,4 & 0 & 0,0 & 1 & 4,3 & \\
\hline $\begin{array}{l}5 \text { a } 6 \text { vezes } \\
\text { por semana }\end{array}$ & 14 & 13,2 & 2 & 14,3 & 3 & 21,4 & 8 & 57,1 & 1 & 7,1 & 0 & 0,0 & \\
\hline Todo dia & 27 & 25,5 & 0 & 0,0 & 0 & 0,0 & 1 & 3,7 & 26 & 96,3 & 0 & 0,0 & \\
\hline $\begin{array}{l}\text { Não } \\
\text { Consome }\end{array}$ & 19 & 17,9 & 1 & 5,3 & 4 & 21,1 & 0 & 0,0 & 1 & 5,3 & 13 & 68,4 & \\
\hline
\end{tabular}

Fonte: Autores (2021).

Os valores de qui-quadrado demonstram que o aumento no consumo de salada crua foi maior entre os indivíduos que relataram não consumir o alimento no período anterior a pandemia. Quanto a frequência do consumo de legumes e verduras cozidos, frutas frescas ou salada de frutas, leite ou iogurte, aa maior elevação foi encontrada entre os indivíduos que já consumiam os grupos alimentares no período pré pandemia. 


\section{Discussão}

A vulnerabilidade e a insegurança alimentar são fatores amplamente identificados entre estudantes. A escassez de recursos financeiros, excesso de atividades e gastos acadêmicos, são condições que contribuem significativamente para o desenvolvimento de diversos desequilíbrios nutricionais (Christensen et al., 2021).

O distanciamento social, adotado como medida de contenção ao coronavírus acarretou uma série de mudanças na rotina e no comportamento de diversas pessoas. Estima-se que essas alterações possam ter sido mais intensas entre estudantes. A adesão ao ensino remoto ou virtual contribuiu para o retorno do estudante a casa dos pais ou familiares, lapidando o comportamento alimentar. Em alguns casos, o retorno ao ambiente familiar significou uma melhoria nos hábitos e no consumo de alimentos de maior qualidade nutricional (Buckland et al., 2021; Huber, Steffen, Schlichtiger, \& Brunner, 2020).

No presente estudo podemos identificar um aumento no consumo de frutas e vegetais, sob a forma de salada de frutas ou saladas cruas e cozidas. O aumento do consumo de frutas pode ser justificado como uma alternativa que supre a necessidade de ter alimentos disponíveis para o consumo sem necessidade de aplicar técnicas de preparo, principalmente nas pequenas refeições ou lanches. O consumo de leites e iogurtes, alimentos que também dispensam preparo, também passou a ser maior no período de distanciamento social. A frequência do consumo de salada crua durante o período pandêmico também foi aumentada, no entanto, as saladas cozidas se mostraram como as de maior preferência.

O aumento do consumo de frutas pode ser resultante do suprimento da necessidade de alimentos com potencial para refeições rápidas, sem necessidade de preparo. Em contraste, o consumo aumentado de saladas cozidas pode sugerir maior dedicação de tempo ao preparo de refeições saudáveis durante a pandemia. Essa conduta pode ser interpretada a partir de duas vertentes: a primeira delas é a preocupação em consumir alimentos saudáveis e evitar o adoecimento. A segunda linha, o desenvolvimento do comer emocional, onde o indivíduo passa a se alimentar ou preparar refeições a partir de suas emoções e não pela fome fisiológica.

A literatura aponta que durante a pandemia o comer emocional se tornou comum entre estudantes, que passaram a buscar alimentos específicos ou elevar o número de refeições em função das emoções (Serin \& Koç, 2020). A relação entre as emoções e o consumo alimentar durante o período de distanciamento social pode levar ao desencadeamento de episódios de compulsão, sendo ainda mais intenso entre indivíduos do sexo feminino e com excesso de peso prévio (Flaudias et al., 2020; Kalkan Uğurlu, Mataracı Değirmenci, Durgun, \& Gök Uğur, 2021).

O comportamento e as escolhas alimentares têm um elo forte com as emoções e, durante o período pandêmico, essa relação sofreu um fortalecimento, principalmente no que se refere aos estudantes. Os estudos apontam para diversas alterações alimentares em função de emoções como tédio, medo e ansiedade. Em estudantes essas emoções foram associadas a compulsão alimentar, repercutindo de diferentes formas. Em alguns casos, esses indivíduos passaram a optar pelo consumo de frutas por serem fáceis e rápidas de consumir e não exigir esforço no preparo(Özden \& Parlar Kiliç, 2021).

Outras pesquisas também desenvolvidas com esse mesmo público apontam como principais motivos para a mudança de hábitos, durante o distanciamento social, a maior disponibilidade de tempo para o preparo e realização de refeições, o retorno ao ambiente familiar e a utilização da alimentação como ferramenta para a melhoria do estado de saúde e fortalecimento do sistema imunológico, levando a maior preferência por alimentos in natura como frutas e vegetais (YILMAZ, Aslan, \& Unal, 2020).

$\mathrm{O}$ ato de comer também carrega diversos aspectos culturais e esse é um dos pontos que contribuíram na modificação dos hábitos alimentares dos estudantes durante o período pandêmico, uma vez que, as refeições passaram a ser realizadas em casa, reforçando o poder de escolha de acordo com hábitos regionais. Uma pesquisa desenvolvida com estudantes dos Estados Unidos da América, identificou que o retorno à casa dos pais, bem como a cultura alimentar familiar foram aspectos envolvidos na modificação das características das refeições. Alguns indivíduos apontaram que a alimentação melhorou, em 
relação ao que era consumido no ambiente universitário, tendo como motivação os hábitos culturais da família (Powell, Lawler, Durham, \& Cullerton, 2021).

\section{Conclusão}

Neste estudo podemos identificar uma melhoria nos hábitos alimentares dos estudantes, sendo caracterizado pela elevação da frequência do consumo de frutas, legumes e verduras, bem como o consumo de leite e iogurtes. Essa modificação do padrão alimentar pode ser resultante da maior disponibilidade de tempo para o preparo de refeições, retorno ao ambiente familiar e a utilização da alimentação como ferramenta essencial para o fortalecimento da saúde e do sistema imunológico.

Destaca-se que o aumento do consumo de frutas, legumes e verduras, se deu, principalmente, entre indivíduos que já consumiam esses grupos de alimentos pelo menos 1 a duas vezes por semana. Dentre o grupo de pessoas que não consumiam saladas cruas, verduras e legumes cozidos, não houve mudanças entre os períodos avaliados. Esse achado desperta para a necessidade de atividades de educação nutricional, incentivando o consumo desses grupos alimentares, bem como os seus benefícios para a saúde.

Diante do exposto, é importante entender as mudanças ocorridas na alimentação do estudante durante a pandemia, bem como, conhecer os fatores relacionados a mudanças de hábitos. Estas informações serão cruciais para o manejo das práticas alimentares, o planejamento da alimentação oferecida no ambiente institucional no período pós pandemia, bem como, estímulo para que pesquisadores se debrucem no aprofundamento do estudo deste grupo.

\section{Referências}

Amatori, S., Zeppa, S. D., Preti, A., Gervasi, M., Gobbi, E., Ferrini, F., \& Sisti, D. (2020). Dietary habits and psychological states during covid-19 home isolation in italian college students: The role of physical exercise. Nutrients, 12(12), 1-17. https://doi.org/10.3390/nu12123660

Auerbach, R. P., Mortier, P., Bruffaerts, R., Alonso, J., Benjet, C., Cuijpers, P., \& Kessler, R. C. (2018). WHO World Mental Health Surveys International College Student Project: Prevalence and distribution of mental disorders. Journal of Abnormal Psychology, 127(7), 623-638. https://doi.org/10.1037/abn0000362

Boukrim, M., Obtel, M., Kasouati, J., Achbani, A., \& Razine, R. (2021). Covid-19 and Confinement: Effect on Weight Load, Physical Activity and Eating Behavior of Higher Education Students in Southern Morocco. Annals of Global Health, 87(1), 1-11. https://doi.org/10.5334/aogh.3144

Brooks, S. K., Webster, R. K., Smith, L. E., Woodland, L., Wessely, S., Greenberg, N., \& Rubin, G. J. (2020). The psychological impact of quarantine and how to reduce it: rapid review of the evidence. The Lancet, 395(10227), 912-920. https://doi.org/10.1016/S0140-6736(20)30460-8

Buckland, N. J., Swinnerton, L. F., Ng, K., Price, M., Wilkinson, L. L., Myers, A., \& Dalton, M. (2021). Susceptibility to increased high energy dense sweet and savoury food intake in response to the COVID-19 lockdown: The role of craving control and acceptance coping strategies. Appetite, 158(July 2020), 105017. https://doi.org/10.1016/j.appet.2020.105017

Christensen, K. A., Forbush, K. T., Richson, B. N., Thomeczek, M. L., Perko, V. L., Bjorlie, K., \& Mildrum Chana, S. (2021). Food insecurity associated with elevated eating disorder symptoms, impairment, and eating disorder diagnoses in an American University student sample before and during the beginning of the $<$ scp $>$ COVID $</$ scp $>-19$ pandemic. International Journal of Eating Disorders, 54(7), 1213-1223. https://doi.org/10.1002/eat.23517

Coakley, K. E., Le, H., Silva, S. R., \& Wilks, A. (2021). Anxiety is associated with appetitive traits in university students during the COVID-19 pandemic. Nutrition Journal, 20(1), 1-9. https://doi.org/10.1186/s12937-021-00701-9

De Pasquale, C., Sciacca, F., Conti, D., Pistorio, M. L., Hichy, Z., Cardullo, R. L., \& Di Nuovo, S. (2021). Relations Between Mood States and Eating Behavior During COVID-19 Pandemic in a Sample of Italian College Students. Frontiers in Psychology, 12(July), 1-8. https://doi.org/10.3389/fpsyg.2021.684195

Donald, S. (2015). Your chi-square test is statistically significant: Now what? Practical Assessment, Research and Evaluation, 20(8), 1-10.

Flaudias, V., Iceta, S., Zerhouni, O., Rodgers, R. F., Billieux, J., Llorca, P.-M., \& Guillaume, S. (2020). COVID-19 pandemic lockdown and problematic eating behaviors in a student population. Journal of Behavioral Addictions, 9(3), 826-835. https://doi.org/10.1556/2006.2020.00053

Huber, B. C., Steffen, J., Schlichtiger, J., \& Brunner, S. (2020). Altered nutrition behavior during COVID-19 pandemic lockdown in young adults. European Journal of Nutrition. https://doi.org/10.1007/s00394-020-02435-6

Kalkan Uğurlu, Y., Mataracı Değirmenci, D., Durgun, H., \& Gök Uğur, H. (2021). The examination of the relationship between nursing students' depression, anxiety and stress levels and restrictive, emotional, and external eating behaviors in COVID-19 social isolation process. Perspectives in Psychiatric Care, 57(2), 507-516. https://doi.org/10.1111/ppc.12703 
Keel, P. K., Gomez, M. M., Harris, L., Kennedy, G. A., Ribeiro, J., \& Joiner, T. E. (2020). Gaining "The Quarantine 15:” Perceived versus observed weight changes in college students in the wake of COVID-19. International Journal of Eating Disorders, 53(11), 1801-1808. https://doi.org/10.1002/eat.23375

Martínez-de-Quel, Ó., Suárez-Iglesias, D., López-Flores, M., \& Pérez, C. A. (2021). Physical activity, dietary habits and sleep quality before and during COVID-19 lockdown: A longitudinal study. Appetite, 158, 105019. https://doi.org/10.1016/j.appet.2020.105019

Ministério da Saúde. (2020). Vigitel Brasil 2019. In Vigitel Brasil 2019 vigilância de fatores de risco e proteção para doenças crônicas por inquérito telefônico: estimativas sobre frequência e distribuição sociodemográfica de fatores de risco e proteção para doenças crônicas nas capitais dos 26 estados .

https://www.saude.gov.br/images/pdf/2020/April/27/vigitel-brasil-2019-vigilancia-fatores-risco.pdf

Özden, G., \& Parlar Kiliç, S. (2021). The Effect of Social Isolation during COVID-19 Pandemic on Nutrition and Exercise Behaviors of Nursing Students. Ecology of Food and Nutrition, 00(00), 1-19. https://doi.org/10.1080/03670244.2021.1875456

Poelman, M. P., Gillebaart, M., Schlinkert, C., Dijkstra, S. C., Derksen, E., Mensink, F., \& de Vet, E. (2021). Eating behavior and food purchases during the COVID-19 lockdown: A cross-sectional study among adults in the Netherlands. Appetite, 157, 105002. https://doi.org/10.1016/j.appet.2020.105002

Serin, E., \& Koç, M. C. (2020). Examination of the eating behaviours and depression states of the university students who stay at home during the coronavirus pandemic in terms of different variables. Progress in Nutrition, 22(4), 33-43. https://doi.org/10.23751/pn.v22i1-S.9780

Sidor, A., \& Rzymski, P. (2020). Dietary choices and habits during COVID-19 lockdown: Experience from Poland. Nutrients, 12(6), 1-13. https://doi.org/10.3390/nu12061657

Yilmaz, H. Ö., Aslan, R., \& Unal, C. (2020). The Effect of the COVID-19 Outbreak on Eating Habits and Food Purchasing Behaviors of University Students. Kesmas: National Public Health Journal, 15(3), 154-159. https://doi.org/10.21109/kesmas.v15i3.3897 